\title{
Effect of Chronic Intermittent Hypoxia (ClH) on Neuromuscular Junctions and Mitochondria in Slow- and Fast-Twitch Skeletal Muscle of Mice - Role of iNOS
}

\section{Laura Isabel Bannow ( $\nabla$ bannow@med.uni-marburg.de )}

Philipps-Universität Marburg: Institute for Anatomy and Cell Biology https://orcid.org/0000-0002-57705963

\section{Gabriel A. Bonaterra}

Department of Cell Biology, Institute for Anatomy and Cell Biologie, Philipps-University Marburg

\section{Mirjam Bertoune}

Department of Cell Biology, Institute for Anatomy and Cell Biology, Philipps-University Marburg

\section{Sabrina Maus}

Department of Cell Biology, Institute for Anatomy and Cell Biology, Philipps-University Marburg

\section{Richard Schulz}

Department of Pulmonary Medicine, HELIOS Dr. Horst Schmidt Clinic, Wiesbaden

\section{Norbert Weissmann}

Justus-Liebig University of Giessen, Excellence Cluster Cardiopulmonary Institute, Universities of Giessen and Marburg Lung Center

\section{Simone Kraut}

Justus-Liebig University of Giessen, Excellence Cluster Cardiopulmonary Institute, University of Giessen and Marburg Lung Center

\section{Ralf Kinscherf}

Department of Cell Biology, Institute for Anatomy and Cell Biology, Philipps-University Marburg

\section{Wulf Hildebrandt}

Department of Cell Biology, Institute for Anatomy and Cell Biology, Philipps-University Marburg

\section{Research}

Keywords: denervation, muscle atrophy, mitochondria, fiber type, oxidative stress, neuromuscular junction, iNOS

Posted Date: November 10th, 2020

DOl: https://doi.org/10.21203/rs.3.rs-102618/v1 
License: (c) (i) This work is licensed under a Creative Commons Attribution 4.0 International License. Read Full License

Version of Record: A version of this preprint was published at Skeletal Muscle on February 12th, 2022. See the published version at https://doi.org/10.1186/s13395-022-00288-7. 


\section{Abstract}

Background: Obstructive sleep apnea (OSA) imposes vascular and metabolic risks through chronic intermittent hypoxia $(\mathrm{ClH})$ and impairs skeletal muscle performance. As studies addressing limb muscles are rare, the reasons for the lower exercise capacity are unknown. We hypothesize that $\mathrm{ClH}$-related morphological alterations in neuromuscular junctions (NMJ) and mitochondrial integrity might be the cause of functional disorders in skeletal muscles.

Methods: Mice were kept under 6-weeks-CIH (alternating 7\% and 21\% $\mathrm{O}_{2}$-fractions every $30 \mathrm{~s}, 8 \mathrm{~h} / \mathrm{d}, 5 \mathrm{~d} / \mathrm{w}$ ) compared to normoxia (NOX). Analyses included neuromuscular junctions (NMJ) postsynaptic morphology and integrity, fiber cross-sectional area (CSA) and composition (ATPase), mitochondrial ultrastructure (transmission-electron-microscopy) and relevant transcripts (qRT-PCR). Beside wildtype (WT) we included inducible-nitric-oxide-synthase knockout mice (iNOS ${ }^{-/}$) to evaluate whether iNOS is protective or risk-mediating.

Results: In WT soleus muscle, ClH vs. NOX reduced NMJ size $(-37.0 \%, p<0.001)$ and length $(-25.0 \%$, $p<0.05)$ together with fiber CSA of type lla fibers $(-14 \%, p<0.05)$ and increased centronucleated fiber fraction $(\mathrm{p}<0.001)$. Moreover, $\mathrm{ClH}$ vs. NOX increased the fraction of damaged mitochondria (1.8-fold, $\mathrm{p}<0.001)$. Compared to WT, iNOS ${ }^{-/}$similarly decreased NMJ area and length with NOX $(-55 \%, p<0.001$ and $-33 \%, \mathrm{p}<0.05$, respectively) or with $\mathrm{ClH}(-37 \%, \mathrm{p}<0.05$ and $-29 \%, \mathrm{p}<0.05)$, however, prompted no fiber atrophy. Moreover, increased fractions of damaged $(2.1$-fold, $p<0.001)$ or swollen $(>6$-fold, $p<0.001)$ mitochondria were observed with $\mathrm{iNOS}^{-/-}$vs. WT under $\mathrm{NOX}$ and similarly under $\mathrm{ClH}$. Both, $\mathrm{ClH}$ - and $\mathrm{iNOS}^{-/-}$ massively upregulated suppressor-of-cytokine-signaling-3 (SOCS3) $>10$-fold. None of these morphological alterations with $\mathrm{ClH}$ - or i $\mathrm{NOS}^{-/-}$were detected in gastrocnemius muscle. Notably, iNOS expression was undetectable in WT muscle, unlike the liver, where it was massively decreased with $\mathrm{CIH}$.

Conclusion: $\mathrm{ClH}$ leads to $\mathrm{NMJ}$ and mitochondrial damage associated with fiber atrophy/centronucleation selectively in slow-twitch muscle of WT. This effect is largely mimicked by iNOS ${ }^{-/}$at NOX (except for atrophy). Both conditions involve massive SOCS3 upregulation likely through denervation. In the absence of muscular iNOS expression in WT, this damage may arise from extramuscular, e.g. motoneuronal iNOS deficiency (through $\mathrm{ClH}$ or knockout) awaiting functional evaluation.

\section{Introduction}

Obstructive sleep apnea (OSA), which describes a repetitive collapse of the upper airways during sleep, causes recurrent episodes of hypopnea or even apnea resulting in chronic intermittent hypoxia $(\mathrm{ClH})$. Over the last two decades, the prevalence of sleep apnea has increased by a double-digit figure [1-5]. In a population-based study among the group of 40 - to 85 -year-old adults, $49.7 \%$ of men and $23.4 \%$ of women suffered from a moderate to severe OSA defined as an apnea-hypopnea index (AHI) $\geq 15$ [6]. Due to the growing rate of obesity that is suspected as one of the strongest causal triggers, a further increase of sleep associated disorders is expected [5]. 
Among various comorbidities, OSA patients often experience muscle fatigue and reduced physical performance [7] resulting in limited daily activities and quality of life. In fact, recent meta-analyses showed that maximum oxygen uptake (aerobic capacity) under cycle ergometer test conditions is significantly reduced in patients with (severe) OSAS $[8,9]$ compared to healthy controls and may be improved by continuous-positive-airway-pressure (CPAP) therapy [10]. The limiting factors of $\mathrm{O}_{2}$-transport or muscle function responsible for an inverse relation between $\mathrm{AHI}$ and aerobic capacity have yet remained unclear, but may not include histomorphological muscle microvascularisation, which was found to be increases at least in the tibialis anterior muscle [11]. While histomorphological data on locomotor muscle with OSA are surprisingly scarce, several studies in striated upper airway muscles have indicated various important alterations such as fiber type grouping $[12,13]$, decreases in fiber crosssectional area [14], muscle fiber atrophy [13], centrally located nuclei [12] or abnormal mitochondrial distribution [15]. Thus, mechanisms underlying functional impairment of locomotor muscles have remained to be assessed. Notably, adequate muscle function is not only dependent on the integrity of muscle fibers themselves but also reliant on intact neuromuscular signal transmission via myelinated motor neuron, neuromuscular junction (NMJ) and postsynaptic sarcolemma [16]. NMJ integrity and plasticity is considered critical for muscular function: While several studies have demonstrated a relationship between increases in pre-and postsynaptic areas and enhanced neuromuscular activity as well as increased fatigue resistance [17-20], age-related changes in NMJ, such as fragmentation or lowered NMJ area, are considered to contribute to sarcopenia [21, 22]. Moreover, investigations on mice lacking antioxidant enzymes ( $\mathrm{SOD}^{-/-}$) or overexpressing uncoupling proteins (UCP1) supported the hypothesis, that oxidative stress may trigger NMJ degenerations which occur in association with mitochondrial dysfunction [23, 24]. Most relevant to OSA, a massive generation of reactive oxygen/nitrogen species (ROS/RNS) and related excessive oxidative and nitrosative stress has been attributed to repetitive nocturnal hypoxia-reoxygenation cycles in analogy to repeated ischemia and reperfusion injuries [25,26]. Indeed, evidence exists in OSA patients for increased production of superoxide, which was normalized under CPAP therapy [27], as well as for a decrease in anti-oxidative capacity $[28,29]$. Moreover, studies in OSA patients as well as in $\mathrm{ClH}$ animal models detected an overexpression of inducible nitric oxide synthase (iNOS) via inflammatory triggers involving NF-KB activation, especially in neuronal or cardiovascular tissues, e.g. activated macrophages [30-32]. iNOS may become a source of massive amounts of nitric oxide (NO, only limited by a lack of substrate or coenzymes) which as a highly reactive free radical forms peroxynitrite and other RNS compromising mitochondrial respiration, cell membrane integrity or insulin signaling [33]. iNOS expression in skeletal muscle is observed in (type 2 fibers) of obese/diabetic adult, but rarely in healthy young subjects, unless exercising, while muscular iNOS expression in rodents was mainly detectable and studied in rats [34].

The present study used $\mathrm{ClH}$ as compared to normoxic control (NOX) as model of OSA in wildtype (WT) mice to investigate the long-term effect of OSA on skeletal muscle histomorphological measures of NMJ integrity, fiber size and composition as well as on transition electron microscopy (TEM) parameters of ultrastructural mitochondria integrity. Moreover, besides WT, we included iNOS-deficient (iNOS ${ }^{-/-}$) mice into this analysis in order to evaluate the putatively risk-mediating role of iNOS within the pro-oxidative or 
-inflammatory stress arising from $\mathrm{ClH}$-exposure. To the best of our knowledge, this is the first report on $\mathrm{ClH}$-induced muscle-specific damage to $\mathrm{NMJ}$, fiber histomorphology or mitochondrial ultrastructure in WT mice locomotor muscle, which unexpectedly is not inhibited by iNOS deficiency, but strikingly mimicked already at NOX.

\section{Materials And Methods}

\section{$\mathrm{CIH}$ - mouse model of OSA:}

The mouse model of OSA was based on the long-term exposure to $\mathrm{CIH}$ as described by Schulz et al. (Schulz et al. 2014). Male C57BL/6J-mice (WT, Charles River Deutschland GmbH, Sulzfeld, Germany) and iNOS-deficient mice (iNOS ${ }^{-/}$; strain B6.129P2-Nos2 ${ }^{\text {tm1 Lau }} / \mathrm{J}$ ) aged 8-10 weeks were exposed to a 6-week $\mathrm{ClH}$ profile ( $8 \mathrm{~h} / \mathrm{d} 5 \mathrm{~d} /$ week) in a PC-controlled normobaric gas chamber which allowed alternating $\mathrm{O}_{2}$ concentration between $7 \%$ and $21 \%$ at cycles of $120 \mathrm{~s}$ corresponding to an $\mathrm{AHI}$ of $30 \mathrm{~h}^{-1}$, i.e. border between moderate to severe sleep apnea. NOX control conditions for age-matched WT and $\mathrm{iNOS}^{-/-}$mice were rendered by the same chamber system flushed with room air $\left(21 \% \mathrm{O}_{2}\right)$. $\mathrm{ClH}$ and control intervention were limited to daytime as mice are active at night. All animals were provided with standard diet and water ad libitum. All mice were weighed prior to intervention and immediately before euthanization.

After 6 weeks of $\mathrm{ClH}$-/control intervention, mice were euthanized and the triceps surae (gastrocnemius, soleus and plantaris muscles) as well as the vastus lateralis muscle carefully removed, immediately shock frozen in liquid nitrogen-cooled isopentane and stored at $-80^{\circ} \mathrm{C}$. Transversal cryosections of $7 \mu \mathrm{m}$ (microtom Hyrax $\mathrm{C} 60$, Carl Zeiss AG, Oberkochen, $-20^{\circ} \mathrm{C}$ ) were obtained for (immuno)histochemistry. Tibia length was carefully determined by a caliper.

\section{Muscle fiber composition, size and centronucleation:}

Muscle fiber types (1, 2a, 2x) were identified via the acid-sensitive myofibrillar ATPase (Adenosine triphosphatase, Sigma-Aldrich Co. LLC, St. Louis, Missouri) staining at pH 4.55 as previously described (Friedmann-Bette et al. 2010) in randomly distributed two to three images taken at 200-fold magnification by the Zeiss Axio Imager.M2 microscope (Carl Zeiss AG; Oberkochen, Germany) after digitalization by the imaging system Axio-Cam HRc/AxioVision (Carl Zeiss $\mathrm{GmbH}$ ). Type-specific fiber cross-sectional area (CSA) was determined by manually encircling each cross-section of at least 100 fibers using standard imaging software ImageJ (Scion Image, National Institutes of Health, Bethesda, USA). In addition, transverse cryosections were stained by hematoxylin and eosin to determine the percentage of centronucleated fibers.

\section{Postsynaptic NMJ morphology and integrity:}

NMJ analyses were based on a-bungarotoxin (BTX) staining in cryosections after fixation with $4 \%$ PFA/PBS (paraformaldehyde/phosphate buffered saline $\mathrm{pH}$ 7.4) for $10 \mathrm{~min}$ and blocking of endogenous peroxidase with $3 \% \mathrm{H}_{2} \mathrm{O}_{2}$. Thereafter, PBS-washed sections were incubated overnight with biotinylated 
BTX (1:500; Invitrogen Eugene, USA) in a humidified chamber, PBS-washed, blocked with $2 \%$ bovine serum albumin (BSA)/PBS, PBS-washed again, and incubated with horseradish peroxidase (HRP)conjugated Streptavidin (Jackson ImmunoResearch Laboratories. Inc., West Grove, USA). 3,3'Diaminobenzidine (DAB) was used as a chromogen substrate. Nuclei were counterstained with Mayer's Hematoxylin (Carl Roth $\mathrm{GmbH}$, Germany).

NMJ were identified in three consecutive, complete cross-sections per muscle from digital images (200fold magnification) obtained by the Zeiss Axio Imager.M2 microscope (Carl Zeiss AG; Oberkochen, Germany) combined with Axio-Cam HRc/AxioVision (Carl Zeiss $\mathrm{GmbH}$ ). The NMJ length and area were determined manually together with the corresponding myofiber CSA and perimeter using ImageJ software (Scion Image, National Institutes of Health). Single NMJ were considered as "fragmented" when the BTX-stained area was divided into several sections. Further calculations included NMJ area per myofiber CSA, NMJ length per myofiber perimeter and the percentage of fragmented NMJ (McLoon et al. 2016). On average, $21 \pm 2(16 \pm 3)$ NMJ were analyzed per soleus or gastrocnemius muscle.

\section{Pre- and postsynaptic NMJ co-staining:}

To analyze the innervation status by double staining of NMJ pre- and postsynapse, every 6th of 60 serial $7 \mu \mathrm{m}$ transverse sections of the vastus muscle (WT: $n=4 \mathrm{NOX}, \mathrm{n}=6 \mathrm{ClH}^{\mathrm{iNOS}}{ }^{-/-}: \mathrm{n}=6 \mathrm{NOX}, \mathrm{n}=6 \mathrm{CIH}$ ) was stained for immunofluorescence with biotin-XX-conjugated BTX (Invitrogen Eugene, USA) and RvAChT (vesicular acetylcholine transporter) antibody (Lee Eiden, Laborchargen-Nr.: bl. 6/97). In detail, after fixation for $10 \mathrm{~min}$ in $4 \%$ PFA/PBS, and blockage by $1 \%$ BSA/PBS for $30 \mathrm{~min}$, sections were incubated overnight at $4{ }^{\circ} \mathrm{C}$ with biotinylated BTX (1:500) or vAChT 80259 (1:1000) in a humidified chamber. The next day, washing was followed by two hours incubation with Alexa Fluor ${ }^{\circledR} 488$ labeled donkey anti-rabbit IgG (1:200, MoBiTec GmbH, Göttingen, Germany) or Cy3-conjugated streptavidin (1:200, Dianova GmbH, Hamburg, Germany) respectively. Finally, slides were mounted using ImmuMount $^{\text {TM }}$, (Fisher Scientific GmbH, Schwerte, Germany) and glass coverslips. Control sections were treated similarly but with either no primary antibody to exclude non-specific staining of secondary antibody. Confocal images were taken by the scanning laser microscope C2 (Nikon GmbH, Düsseldorf, Germany) using the software NIS-Elements AR 4.30.01 (Laboratory Imaging). Each NMJ was scanned in a 630-fold magnification at $250 \mathrm{~Hz}$ with an image size of $1024 \times 1024$ pixels. Subsequently, Fiji software (National Institute of Health, Bethesda, USA) was used for morphometrical analysis, determining the BTX and vAChT immunolabeled areas as well as their overlap area.

\section{Transmission electron microscopy (TEM):}

Mitochondrial ultrastructure of soleus and gastrocnemius muscle was evaluated using TEM as previously described [35]. Images were taken at 10.000-fold magnification and ten random pictures from both muscles of each mouse were analyzed using ImageJ software (Scion Image, National Institutes of Health, Bethesda, USA). Mean mitochondrial size was determined by manually encircling all identified mitochondria. Alterations on mitochondrial architecture were assessed by the categorization "normal" or "damaged" as follows: Mitochondria showing a loss of more than $50 \%$ of the cristae or a disruption of 
more than $50 \%$ of the outer membrane were assigned to "damaged" or otherwise to "normal". The results were given as the percentage of the total number of mitochondria. Moreover, the percentage of swollen mitochondria and mitochondria containing multi-lamellar bodies was determined.

\section{Quantitative RT-PCR:}

The extraction of RNA was performed using peqGOLD Isolation Systems TriFast ${ }^{\mathrm{TM}}$ (PEQLAB Biotechnologie $\mathrm{GmbH}$, Erlangen, Germany) according to the manufacturer's instructions. RNA quality (OD260nm / OD280nm = 1.7 to 2.0) and concentration were determined using the NanoDrop 2000c spectrophotometer (Thermo Scientific, Schwerte, Germany). RNA integrity was confirmed by lab-on-a-chip technology, using an RNA 6000 NanoChip kit on an Agilent 2100 Bioanalyzer (Agilent Technologies, Waldbronn, Germany). Total RNA $(0.7 \mu \mathrm{g})$ was then treated with 1 unit DNAse (Thermo Scientific, St. Leon-Rot, Germany; 30 min, $37^{\circ} \mathrm{C}$ ). Thereafter, reverse transcription of RNA was carried out with $500 \mathrm{ng}$ oligo (dT) 12- 18 primer, 20 units of the Affinity Script multiple temperature cDNA synthesis (Agilent) and 24 units of Ribo Lock ${ }^{\mathrm{TM}}$ RNAse inhibitor (Fermentas; $1 \mathrm{~h}, 42^{\circ} \mathrm{C}$ ) and $4 \mathrm{mM}$ dNTP-Mix. Quantitative reverse transcriptase polymerase chain reaction (qRT-PCR) was performed in duplicate using the QuantiTect/primerAssays from QIAGEN GmbH (Hilden, Germany). To confirm the primer specificity and the presence of a single amplicon, a melting curve $\left(55-95^{\circ} \mathrm{C}\right)$ of the amplified product was performed. For amplification and data analysis the Mx3005 $\mathrm{P}^{\mathrm{TM}}$ QPCR System (Stratagene) was used. For each sample, the relative amount was calculated by linear regression analysis from their respective standard curves which was generated from a pool of cDNA. Among the transcripts Actb, Gapdh and Tbp, Tbp was identified by the NormFinder software as the most stable reference gene for RNA normalization of gene of interest (additional file 1).

\section{Statistics:}

Data are presented as means \pm standard error of the mean (SEM). Differences between $\mathrm{ClH}$ and NOX or iNOS $^{-/-}$and WT were detected by ANOVA and Student's t-test (post-hoc) or, in case of not normally distributed data, by the Kruskal-Wallis ANOVA and Dunn's post-hoc test. A $p<0.05$ was considered as statistically significant. For all statistical procedures SigmaPlot 14.0 software (Systat Software, Inc, Chicago, USA) was used.

\section{Results}

\section{Body weight:}

\section{Effect of $\mathrm{ClH}$ in WT}

In WT mice, 6 weeks of ClH compared to NOX significantly reduced the pre- to post-interventional gain in body weight, i.e., the weight gain between 2 and 3.5 months of age (starting from a $9.1 \%$ higher baseline) (Table 1). 
Under NOX conditions, iNOS ${ }^{-/-}$mice showed a significantly lower weight gain compared to WT (Table 1), notably, starting from by $10.7 \%(p<0.001)$ higher pre-interventional body weight than WT mice. When exposed to $\mathrm{ClH}$, iNOS ${ }^{-/-}$mice even showed a weight loss and thus differed significantly from NOX conditions compared to NOX (Table 1).

Table 1

Weight (g) and weight changes (\%) of WT and iNOS ${ }^{-/-}$mice pre- to post-intervention. Values are given as mean + SEM, $n=6-14$ animals per group. * $p<0.05$, significance between $\mathrm{ClH}$ and NOX; \#\# $\mathrm{p}<0.01$, \#\# $\mathrm{p}<0.001$, significance between WT and $\mathrm{iNOS}^{-/-}$.

\begin{tabular}{|lllll|}
\hline & $\begin{array}{l}\text { WT NOX } \\
(\mathbf{n = 1 1})\end{array}$ & $\begin{array}{l}\text { WT ClH } \\
(\mathbf{n = 1 4})\end{array}$ & $\begin{array}{l}\text { iNOS }^{-/-} \text {NOX } \\
(\mathbf{n = 6})\end{array}$ & $\begin{array}{l}\text { iNOS }^{-/-} \mathrm{CIH} \\
(\mathbf{n = 8})\end{array}$ \\
\hline Pre-intervention weight (g) & $21.9 \pm 0.5$ & $23.9 \pm 0.5^{*}$ & $24.8 \pm 0.7^{\# \# \#}$ & $26.0 \pm 0.9$ \\
\hline Post-intervention weight (g) & $26.4 \pm 0.5$ & $25.9 \pm 0.3$ & $25.5 \pm 0.7$ & $24.1 \pm 0.3$ \\
\hline Weight change (\%) & $21.1 \pm 3.7$ & $9.0 \pm 2.0^{*}$ & $2.9 \pm 3.0^{\# \#}$ & $-7.4 \pm 0.8^{*}$ \\
\hline
\end{tabular}

\section{Fiber morphology and fraction in soleus muscle:}

\section{Effect of $\mathrm{ClH}$ in WT}

The mean CSA covering all fiber types in soleus muscle did not significantly differ between $\mathrm{ClH}$ intervention and NOX in WT (Fig. 1A-B, E left), though it correlated significantly and positively with the weight gain $(r=0.41 ; p=0.047)$ in WT of both interventional groups. A fiber type-specific analysis showed, that $\mathrm{CIH}$ compared to NOX led to a significant decrease in fiber CSA exclusively of type $2 \mathrm{a}$ fibers by $13.5 \%$ $(p<0.05)$ (Fig. 1E, right). Thereby, $\mathrm{ClH}$ as compared to NOX resulted in an increase in type 1 fiber fraction $(37 \%$ vs. $31 \% ; p<0.01)$ in association with a decrease in type $2 a$ fiber fraction $(44 \%$ vs. $53 \% ; p<0.001)$ at unchanged type $2 x$ fiber fraction (Fig. 1A-B, G).

A parallel CSA-specific analyses of fiber distribution showed that $\mathrm{ClH}$ compared to NOX significantly increased the fraction of small fibers $\left(\mathrm{CSA}<800 \mu \mathrm{m}^{2}\right)$ in soleus muscle (Fig. 2).

Moreover, there was a significantly higher fraction of centronucleated fibers with $\mathrm{ClH}$ compared to NOX ( $1.5 \%$ vs. $0.2 \% ; p=0.01$ ) in WT soleus muscle, as counted in HE stained sections (Fig. 3 , left). A significant inverse correlation was found between the fraction of centronucleated fibers and the CSA of the fiber population in WT undergoing $\mathrm{ClH}$ or NOX $(r=-0.417 ; p=0.043)$.

Effect of iNOS w/o ClH: In both conditions, NOX and $\mathrm{ClH}$, iNOS ${ }^{-/-}$compared to WT was without any significant effect on fiber CSA of the total fiber population or that of fiber type 1, 2a or 2x. In iNOS ${ }^{-/-}$mice, $\mathrm{CIH}$ as compared to NOX caused no significant changes in CSA of fibers in total or of type (Fig. 1F), while $\mathrm{ClH}$-related changes in fiber composition partly resembled those in WT (Fig. 1A, C, H): ClH compared to NOX in iNOS ${ }^{-/-}$mice led to a decrease in type 2 a fiber fraction $(45 \%$ vs. $51 \% ; p<0.05)$ and an associated 
non-significant increase in type 1 fiber fraction (39\% vs. $35 \%$ ) at unchanged type $2 x$ fiber fraction (Fig. 1C, $D, H)$. Concerning the centronucleation, no significant difference was detected between the two genotypes or between $\mathrm{ClH}$ and NOX in iNOS ${ }^{-/-}$mice (Fig. 3).

\section{Fiber morphology and fraction in gastrocnemius muscle:}

In contrast to soleus muscle, the gastrocnemius muscle mostly consisting of fiber type $2 x$ revealed no significant effect of $\mathrm{ClH}$ vs. NOX and $\mathrm{NNOS}^{-/-}$vs. WT or their combination with regard to CSA (additional file 2) or percentage of central nuclei (additional file 3).

\section{NMJ morphology/integrity in soleus muscle:}

\section{Effect of $\mathrm{ClH}$ in WT mice}

In soleus muscle of WT mice BTX-staining of NMJ showed, that CIH compared to NOX led to a significantly by $37 \%$ smaller postsynaptic NMJ area (Fig. 4A-C, left). When calculating NMJ area relative to fiber CSA, (Fig. 4D, left), the ClH compared to NOX significantly reduced postsynaptic NMJ size. An alternative normalizing of $\mathrm{NMJ}$ length to fiber perimeter similarly decreased $\mathrm{NMJ}$ size with $\mathrm{CIH}$ compared to NOX (Fig. 4E, left). No sigificant ClH-related change occurred regarding the fraction of fragmented NMJ in WT mice (Fig. 4F, left, G).

\section{Effect of iNOS ${ }^{-/-}$w/o ClH}

In comparison to $\mathrm{WT}$, iNOS ${ }^{-/-}$mice demonstrated a significantly by $55 \%$ diminished $\mathrm{NMJ}$ area under the condition of NOX (Fig. 4C), with this effect being significant also upon normalization of postsynaptic NMJ area for fiber CSA (Fig. 4D) or, alternatively, of postsynaptic NMJ length for fiber perimeter (Fig. 4E). $\mathrm{ClH}$ compared to NOX intervention in $\mathrm{NOS}^{-/-}$mice led to an additional decrease in NMJ area (in absolute terms) by trend (Fig. 4C, right), however, this effect reached significance when normalizing postsynaptic NMJ area for fiber CSA (Fig. 4D, right) or, alternatively, postsynaptic NMJ length for fiber perimeter (Fig. 4E, right). Notably, a strikingly higher percentage of NMJ fragmentation was observed selectively with iNOS ${ }^{-/-}$as compared to WT under both conditions (Fig. 4F, right, H).

\section{NMJ morphology/integrity in gastrocnemius and vastus muscle:}

\section{Gastrocnemius muscle}

In contrast to soleus muscle, gastrocnemius muscle in WT mice showed a significantly increased NMJ size with $\mathrm{ClH}$ as compared to $\operatorname{NOX}(72 \%, \mathrm{p}<0.05$, additonal file 4). However, this difference was abolished when normalizing postsynaptic NMR area for fiber CSA. Moreover, unlike the soleus muscle, the gastrocnemius muscle in iNOS ${ }^{-/-}$mice revealed no significant alterations in postsynaptic NMJ area, and this was also true for both above-mentioned normalization of NMJ area or length for fiber CSA or perimeter, respectively. 


\section{Vastus muscle}

For further evaluation of functional NMJ integrity, double fluorescent staining was used in vastus muscle to quantify the area of the NMJ presynaptic nerve terminal (vACHT-antibodies, Fig. 5A, D), of postsynaptic NMJ (BTX, Fig. 5B, E) and that of their coupling (overlay, Fig. 5C, F). In line with the findings in soleus (but not in gastrocnemius) muscle, there was a significant diminution by $27.9 \%(p<0.01)$ of postsynaptic $\mathrm{NMJ}$ in iNOS ${ }^{-/-}$compared to WT under conditions of NOX (Fig. 5G), whereas $\mathrm{ClH}$-effects compared to NOX were absent in WT or iNOS ${ }^{-/-}$mice. The presynaptic terminal, defined as the $\mathrm{VACHT}$ immunoreactive area, remained resistant against $\mathrm{ClH}$ - or genotype related effects $(5 \mathrm{H})$. The resulting percentage overlay area, a measure of NMJ integrity, was not significantly affected by $\mathrm{ClH}$-intervention or $\mathrm{iNOS}^{-/-}$(Fig. 5I).

\section{Mitochondrial ultrastructure in soleus muscle:}

The following ultrastructural mitochondrial abnormalities were quantified in soleus muscle of WT exposed to NOX (Fig. 6A) or ClH (Fig. 6B) as well as in iNOS ${ }^{-1-}$ mice in NOX (Fig. 6C) or ClH (Fig. 6D): Swollen matrix (Fig. $6 \mathrm{H}$ ), disruption of the outer mitochondrial membrane (Fig. 6I), a complete loss of internal architecture (Fig. 6J) and mitochondria with multi-lamellar bodies (Fig. 6K).

\section{Effect of $\mathrm{ClH}$ in WT}

The percentage of damaged mitochondria, classified as $<50 \%$ filled with cristae, was significantly 1.8 fold higher in $\mathrm{CIH}$ vs. NOX (Fig. 6E, left), while an increase in percentage swollen mitochondria with $\mathrm{CIH}$ did not reach significances (Fig. 6F, left). The difference between $\mathrm{CIH}$ vs. NOX regarding the percentage mitochondria containing multi-lamellar bodies did not reach significance $(12.8 \%$ vs. $6.6 \%$; $p>0.05)$.

\section{Effect of iNOS ${ }^{-/-}$w/o ClH}

Somewhat reminiscent of $\mathrm{ClH}$ vs. NOX effects in WT, iNOS ${ }^{-/-}$compared to WT mice at NOX conditions revealed a significant 2.1-fold increase of damaged mitochondria (Fig. 6E). Moreover, percentage of swollen mitochondria in iNOS ${ }^{-/-}$vs. WT mice was significantly increased under both conditions (NOX > 6fold; $\mathrm{ClH}>5$-fold) (Fig. 6F).

\section{Mitochondrial ultrastructure in gastrocnemius muscle:}

In gastrocnemius muscle, the abovementioned mitochondrial alterations were observed neither with $\mathrm{ClH}$ vs. NOX in WT nor with iNOS ${ }^{-/-}$of either condition (additional files 5-6).

\section{Correlations between NMJ fiber and mitochondrial morphology:}

In soleus muscle the NMJ area was significantly correlated with the percentage of damaged mitochondria $(r=0.584, p=0.002)$ as well as with the ratio between type 1 and type $2 a$ fibers $(r=0.397, p$ 
$=0.05)($ Table 2$)$.

Table 2

Correlations of NMJ area and fragmented NMJ, percentage of damaged mitochondria, myofiber CSA and fiber type ratio $(1 / 2 a)$ in soleus muscles, $n=5-8$ animals per group.

Soleus muscle ( $n=5-8$ animals per group)

\begin{tabular}{|lllll|}
\hline & & NMJ area $\left(\mu \mathrm{m}^{2}\right)$ & Fragmented NMJ (\%) & Fiber type ratio; 1/2a \\
\hline Damaged mitochondria (\%) & $\mathrm{r}$ & -0.710 & 0.584 & 0.394 \\
\cline { 2 - 5 } & $\mathrm{p}$ & 0.000 & 0.002 & 0.051 \\
\hline NMJ area $\left(\mu \mathrm{m}^{2}\right)$ & $\mathrm{r}$ & -0.642 & -0.575 \\
\cline { 2 - 4 } & $\mathrm{p}$ & 0.001 & 0.003 \\
\hline Fragmented NMJ (\%) & $\mathrm{r}$ & & 0.396 \\
& $\mathrm{p}$ & & 0.050 \\
\hline
\end{tabular}

\section{Transcripts of iNOS, SOCS3, SOD2 and pro-/antiapoptotic markers:}

Notably, mRNA expression of iNOS was undetectable in soleus (as well as in gastrocnemius) muscle of WT mice undergoing NOX or $\mathrm{CIH}$ intervention (Table 3). However, importantly, an iNOS expression was well detectable in the liver of WT mice, where it decreased significantly and massively by factor 0.12 (Table 3) after $\mathrm{ClH}$-treatment. The absence of iNOS expression was proven in the liver and soleus (and gastrocnemius) muscle of iNOS ${ }^{-/-}$mice.

Importantly, in WT mice, SOCS3 expression was found to be $>10$-fold increased with $\mathrm{ClH}$ compared to NOX (Table 3). Similarly, iNOS ${ }^{-/-}$prompted a SOCS3 upregulation that was $>10$-fold in NOX and $>4$-fold in $\mathrm{ClH}$ as compared to WT at NOX (Table 3).

Moreover, in WT mice, $\mathrm{ClH}$ compared to NOX led to an almost 50\% decrease in mtSOD mRNA expression in soleus muscle. Similarly, iNOS ${ }^{-1-}$ resulted in a $33 \%$ and a $45 \%$ decrease in SOD2 expression in soleus with $\mathrm{NOX}$ and $\mathrm{ClH}$, respectively (Table 3).

Furthermore, the screening for apoptotic markers (BAX, BCL2, caspase 3) showed neither $\mathrm{ClH}$ - nor iNOS ${ }^{-1}$ -related changes in soleus muscle (Table 3). 
Table 3

Relative gene expression in soleus muscle and liver of WT and $\mathrm{iNOS}^{-/-}$mice.

\begin{tabular}{|c|c|c|c|c|c|c|}
\hline & iNOS & socs3 & SOD2 & BAX & BCL2 & Caspase 3 \\
\hline \multicolumn{7}{|c|}{ Soleus muscle } \\
\hline WT NOX & 0.00 & 1.00 & 1.00 & 1.00 & 1.00 & 1.00 \\
\hline WT ClH & 0.00 & 10.89 & 0.53 & 1.10 & 1.14 & 0.90 \\
\hline $\mathrm{iNOS}^{-/-}$NOX & 0.00 & 10.45 & 0.75 & 1.23 & 1.01 & 1.09 \\
\hline $\mathrm{iNOS}^{-/-} \mathrm{ClH}$ & 0.00 & 4.43 & 0.55 & 0.98 & 1.07 & 0.79 \\
\hline \multicolumn{7}{|l|}{ Liver } \\
\hline WT NOX & 1.00 & & & & & \\
\hline WT CIH & 0.12 & & & & & \\
\hline
\end{tabular}

\section{Discussion}

Using long-term $\mathrm{ClH}$ exposure in mice as a model of OSA, the present study shows for the first time that $\mathrm{CIH}$ compared to NOX causes damage of potential functional relevance in 'red' (soleus) but not in 'white' (gastrocnemius) muscle. This comprises a reduction in area, in length and, by trend, in integrity of postsynaptic NMJ as well as in size (CSA) and fraction of type 2a fibers (at higher type-1 fiber fraction). Moreover, these changes were associated with considerable mitochondrial damage, which showed a significant correlation to (loss in) NMJ area $(r=-0.71, p<0.001)$ and were, again, limited to soleus muscle, while gastrocnemius revealed no significant mitochondrial damage.

The present study furthermore included $\mathrm{iNOS}^{-/-}$mice into this analysis of $\mathrm{CIH}$ vs. NOX effects on skeletal muscle, in order to test the hypothesis that iNOS deficiency may at least in part protect against a proinflammatory/-oxidative effect through $\mathrm{ClH}$, i.e. hypoxia-reoxygenation stress leading to ROS generation from various sources [36]. Contrary to expectation, our data demonstrate that, compared to WT, iNOS ${ }^{-/-}$ by itself (i.e. under NOX conditions) also leads to highly significant postsynaptic NMJ area reduction and fragmentation in combination with mitochondrial damage and swelling, which surprisingly resemble and exceed those observed with $\mathrm{CIH}$ in WT mice. Notably, under the conditions of iNOS deficiency, $\mathrm{ClH}$ stress is able to further aggravate the damage at least in terms of a further reduction in postsynaptic NMJ area or length after normalization for fiber CSA or perimeter, respectively. The similarity between $\mathrm{CIH}$ (compared to NOX in WT) and iNOS ${ }^{-/-}$(compared to WT in NOX) was limited to NMJ and mitochondrial damage, while decreases in fiber CSA (including its correlation to NMJ) and centronucleation observed with $\mathrm{ClH}$ vs. NOX in WT were absent in $\mathrm{NOS}^{-/-}$mice, i.e. they revealed no atrophy despite signs of denervation. 
As another striking similarity, we found a > 10-fold increase in SOCS3 expression with CIH vs. NOX in WT as well as with iNOS ${ }^{-/-}$vs. WT at NOX in (pooled samples of) soleus muscle. Available evidence qualifies SOCS3 as a candidate to mechanistically link mitochondrial damage to NMJ deterioration: SOCS3 upregulation has been demonstrated as an early event after skeletal muscle denervation by sciatic nerve transection [37]. This obviously occurs in response to local inflammatory signals, especially via IL6 which by itself, i.e. without denervation, causes upregulation of SOCS3 and of E3-ligases (atrogin-1, MURF-1) together with fiber atrophy all of which is abrogated by IL6 inhibition. At the same time, SOCS3 overexpression has been shown to cause mitochondrial damage like swelling or disruption in tibialis anterior muscle, which is reminiscent of what was presently observed in soleus muscle but not in gastrocnemius muscle. SOCS3 overexpression was, furthermore, associated with inhibited expression of mitochondrial genes, which included Smtck and SIc25a3 [38], but may also comprise mtSOD, which was presently found to be downregulated. As an inhibitor of leptin and insulin signaling, increased muscular SOCS3 expression has been suggested as a major contributor to mitochondrial dysfunction, impaired fatty acid oxidation, as associated with aging, metabolic syndrome and inflammation [39-41]. These severe metabolic effects in combination with previous evidence that SOCS3 overexpression dilates the sarcoplasmatic reticulum, dislocates and inhibits calcineurine (colocalized with SOCS3) and reduces skeletal muscle energy expenditure, oxygen uptake and activity [38] may contribute to muscle fiber atrophy as observed in case of $\mathrm{ClH}$. While we found no evidence for increased apoptosis signals, a SOCS3 upregulation appears to be associated with impaired regenerative stem cell function in elderly humans [39] and may potentially play a role in the increased centronucleation presently observed with $\mathrm{ClH}$.

Moreover, the moderate decrease in $\mathrm{mtSOD}$ expression presently observed in soleus muscle with $\mathrm{ClH}$ and, to a lesser extent, with iNOS (NOX or $\mathrm{ClH}$ ) might also play a role in fiber atrophy and mitochondrial deterioration: Sod1 ${ }^{-/-}$mice, used as a murine model of neuromuscular impairment in age-related muscle atrophy (sarcopenia), exhibit reduction in myofiber CSA of type lla fibers [42, 43]. Reduced CSA of type lla fibers was, indeed, presently observed in association with the most marked mtSOD decrease (ca 50\%), lower weight gain and alterations in mitochondrial ultrastructure and NMJ morphology. In addition, the observed shift in fiber metabolic phenotype, i.e. an increased type 1 at a decreased type 2 fiber fraction with both $\mathrm{CIH}$ (vs. WT) and iNOS ${ }^{-/-}$(vs. WT at NOX) might be attributed to decreased mtSOD expression, rather than to SOCS3 upregulation which decreases oxidative fiber characteristics [38]. Deficiency in mtSOD, representing impaired antioxidant defense, may also be involved in a remarkable number of agerelated features which may originate from a loss of fast motoneurons followed by a reinnervation of slow motoneurons [44]. In the present study, fiber type ratio (type 1/type 2a) was significantly correlated with NMJ fragmentation and inversely correlated with NMJ size, pointing at a role of reinnervation in the fiber type shift.

Thus, our observations with $\mathrm{ClH}$ may display some analogies to age-related neuromuscular deterioration involving SOCS3 and mtSOD. They are in line with previous studies in other rodent $\mathrm{ClH}$ models, revealing 
downregulation of mtSOD/SOD2 via downregulation of HIF-2a [45] and clinical observation of lower plasma CuZnSOD/SOD1 in OSA patients [46].

Our $\mathrm{ClH}$-based OSA mouse model is, however, at variance with biopsy studies in OSA patients, which revealed no changes in tibialis anterior muscle fiber size compared to controls [11] or showed even enlarged diameters of type $2 a$ fibers in quadriceps femoris at unaltered fiber type composition [47]. One should, however, bear in mind, that the $\mathrm{CIH}$ mouse model does not mimick certain OSA-inherent factors like sways in intrathoracic pressure and blood $\mathrm{pCO}_{2}$ as well as ventilatory overshoots but at the same time involves more severe $\mathrm{O}_{2}$-desaturation without airway obstruction. Also the genetic background of mice may affect the degree of atrophy [48].

Even more important, our data provide first evidence for a strikingly differential effect of $\mathrm{ClH}$-exposure between soleus and gastrocnemius i.e. (mixed) 'red' and 'white' muscles, that has to be taken into account in translational studies. Indeed, in contrast to soleus muscle, gastrocnemius muscle revealed neither mitochondrial damage nor NMJ alterations (rather enlargement than shrinkage) with $\mathrm{ClH}$. The differential exertion profiles between the postural soleus muscle (remaining recruited throughout during quiet standing) and the locomotor gastrocnemius muscle (providing fast forceful contractions) [49] may impact these muscle-specific findings in $\mathrm{CIH}$ and $\mathrm{iNOS}^{-/-}$mice and have likewise been implicated in massive muscle- (fiber-) specific differences in muscle aging or neurodegenerative disease [50-52].

However, our findings of compromised mitochondrial ultrastructure and gene expression in soleus muscle of $\mathrm{CIH}$-mice may be in line with those in human palate muscle (a primary research focus within OSA pathophysiological), showing abnormal mitochondrial function and organization [53]. The observed close positive correlation of the fraction of damaged mitochondria to fragmented NMJ (or inverse correlation to NMJ area) reveals no clue for cause-effect relationship. As a first assumption, mitochondria-derived oxidative stress during $\mathrm{ClH}$ (hypoxia-reoxygenation stress) may compromise $\mathrm{NMJ}$ [54] acting in combination with other ROS sources like upregulated NOX2, as reported for the presently used $\mathrm{ClH}$ mouse model [55]. However, importantly, iNOS ${ }^{-/-}$at NOX largely mimicked the ClH effects, i.e. $\mathrm{NMJ}$ and mitochondrial damage together with SOCS3 up- and mtSOD downregulation and was despite the fact that iNOS mRNA expression in soleus or gastrocnemius muscles in WT was neither detectable with NOX nor with $\mathrm{ClH}$ exposure. Since, in contrast, WT mice revealed a hepatic iNOS expression, which was massively and significantly reduced ( $>8$-fold) with $\mathrm{ClH}$ compared to NOX, it is reasonable to assume that iNOS deficiency outside the skeletal muscle conveys both, the $\mathrm{ClH}$ and iNOS ${ }^{-/-}$effects. Thereby the NMJ damage, similarly observed with both these condition, strongly points towards an iNOS deficiency in peripheral nerves (i.e. in perikaryon of motoneurons or Schwann cells) as a cause of NMJ damage, though myeloid iNOS expression may also become muscle-protective [56]. Peripheral nerve injury may dramatically upregulate the low constitutive iNOS expression in Schwann cells, and iNOS deletion may result in smaller regenerating myelinated fibers and delayed reinnervation of muscle $\mathrm{NMJ}$ distal to the injury [57]. In fact, peripheral nerve dysfunction in patients suffering from OSA appears to be an early event [58], and denervation may precede muscular dysfunction, as suggested for human upper airway 
muscles [37, 59, 60] and supported by increased sarcolemmal N-CAM staining [15]. To date, no corresponding neuromuscular data exist for human locomotor muscle with OSA. However, they are needed to evaluate functional relevance of these alterations and to separate OSA-specific effects on $\mathrm{NMJ}$, mitochondria, metabolism and related fiber dysfunction from the processes of aging and degenerative diseases $[22,61,62]$. Notably, the age of mice presently under test (four months) corresponded to early human adulthood (20-30 years) [63].

The conclusion that iNOS expression (outside skeletal muscle) may be neuro-protective and relevant for 'red' (aerobic) muscle function may be somewhat counterintuitive, as iNOS upregulation is resulting in a boost of NO, that is antimicrobial or antitumoral but also cytotoxic to normal tissue [64]. Furthermore it is causally implicated e.g. in insulin resistance and diabetes. Nonetheless, in humans a basal NO production rate (rendered mostly but not exclusively by nNOS and eNOS) is physiologically required (reviewed by [34]). There is evidence that NO may convey physiological oxidative signals [65] and a certain production by iNOS is required for neuroprotective antioxidative defense [66], e.g. through the ROS scavenging function of NO $[64,66]$. Data on skeletal muscle tissue are scarce, however, it was reported that iNOS deficiency leads to mitochondrial damage in myocardial dysfunction (adriamycin-based mouse model). Interestingly, this effect was abrogated by overexpression of $\operatorname{mtSOD}[67,68]$, which presently was found to be downregulated with both, $\mathrm{ClH}$ or iNOS deletion.

As a limitation, this study includes no functional data regarding NMJ and skeletal muscle to challenge the relevance of morphological alterations. Moreover, our mouse model involved a limited $\mathrm{ClH}$ exposition of 5 days per week, which may allow adaptive or protective effects of 2 normoxic days per week. Nonetheless a previous study showed, that the pathophysiological changes of the clinical OSA, such as arterial hypertension, are accurately reflected by the here used $\mathrm{CIH}$ mouse model [55].

\section{Conclusion}

In summary, this is the first study to demonstrate $\mathrm{CIH}$ as a model of moderate to severe OSA triggers NMJ and mitochondrial damage accompanied by fiber atrophy in slow-twitch muscle of WT mice, all of which may contribute to reduced exercise (aerobic) capacity in patients suffering from OSA. We furthermore demonstrate that iNOS deficiency, rather than yielding protection of skeletal muscle against $\mathrm{ClH}$ stress, leads to similar structural impairments of NMJ and mitochondria under normoxia and might contribute to the $\mathrm{ClH}$ effects in WT, putatively through compromised innervation.

\section{Abbreviations}

\section{OSA - obstructive sleep apnea}

\section{$\mathrm{ClH}$ - chronic intermittent hypoxia}


NMJ - neuromuscular junction

NOX - normoxia

CSA - cross-sectional area

ATP - adenosine triphosphate

TEM - transmission-electron-microscopy

WT - wildtype

iNOS - inducible-nitric-oxide-synthase

SOCS3 - suppressor-of-cytokine-signaling-3

AHI - apnea-hypopnea index

CPAP - continuous-positive-airway-pressure

UCP - uncoupling protein

ROS - reactive oxygen species

RNS - reactive nitrogen species

NO - nitric oxide

BTX - a-bungarotoxin

PFA - paraformaldehyde 
PBS - phosphate buffered saline

HRP - horseradish peroxidase

BSA - bovine serum albumin

DAB - 3,3'-Diaminobenzidine

vAChT - vesicular acetylcholine transporter

Actb - actin beta

GADPH - glyceraldehyde-3-phosphate dehydrogenase

TBP - TATA-box binding protein

SEM - standard error of the mean

mtSOD - mitochondrial superdioxide dismutase

BAX - BCL-2-associated X protein

BCL2 - b-cell lymphoma 2

MURF-1 - Muscle RING-finger protein-1

sMtCK - sarcomeric mitochondrial creatine kinase

Slc25a3 - Solute Carrier Family 25 Member 3

nNOS - neuronal-nitric-oxide-synthase 


\section{eNOS - endothelial-nitric-oxide-synthase}

\section{Declarations}

\section{Ethics approval and consent to participate}

Animal experiments were approved by the regional board (RP Giessen, Hesse, Germany; Az: V 54-19 c 20 $15 \mathrm{~h} 01 \mathrm{GI}$ 20/10 Nr. 84/2011) in accordance with the German animal welfare law and the European legislation for the protection of animals used for scientific purposes $(2010 / 63 / \mathrm{EU})$.

\section{Consent for publication}

Not applicable

\section{Availability of data and materials}

The datasets used and/or analyzed during the current study are available from the corresponding author on reasonable request. Materials used in this study are commercially available.

\section{Competing interests}

The authors declare that they have no competing financial interests.

\section{Funding}

This work was supported by grants from the von-Behring-Röntgen-Stiftung (Project 580071).

\section{Authors' contributions}

RK and WH conceived, managed and supervised the project, designed experiments and obtained funding. RS and NW contributed to the study design and edited the manuscript. LB and SM performed experiments and analyzed data with contributions from GB, MB and SK. LB and WH wrote the manuscript with input from all authors. The authors read and approved the final manuscript.

\section{Acknowledgements}

The authors gratefully acknowledge the expert laboratory assistance of Claudia Keppler, Michael Dreher, Steffi Zügel and Irmgard Dammshäuser.

\section{References}

1. T. Young, P. E. Peppard, and S. Taheri, “Excess weight and sleep-disordered breathing," Journal of applied physiology (Bethesda, Md. : 1985), vol. 99, no. 4, pp. 1592-1599, 2005. 
2. T. Young, P. E. Peppard, and D. J. Gottlieb, “Epidemiology of obstructive sleep apnea: A population health perspective," American journal of respiratory and critical care medicine, vol. 165, no. 9 , pp. 1217-1239, 2002.

3. T. Young, M. Palta, J. Dempsey et al., "The occurrence of sleep-disordered breathing among middleaged adults," The New England journal of medicine, vol. 328, no. 17, pp. 1230-1235, 1993.

4. A. B. Newman, G. Foster, R. Givelber et al., "Progression and regression of sleep-disordered breathing with changes in weight: The Sleep Heart Health Study," Archives of internal medicine, vol. 165, no. 20, pp. 2408-2413, 2005.

5. P. E. Peppard, T. Young, J. H. Barnet et al., "Increased prevalence of sleep-disordered breathing in adults," American journal of epidemiology, vol. 177, no. 9, pp. 1006-1014, 2013.

6. R. Heinzer, S. Vat, P. Marques-Vidal et al., "Prevalence of sleep-disordered breathing in the general population: The HypnoLaus study," The Lancet Respiratory Medicine, vol. 3, no. 4, pp. 310-318, 2015.

7. R. D. Chervin, "Sleepiness, fatigue, tiredness, and lack of energy in obstructive sleep apnea," Chest, vol. 118, no. 2, pp. 372-379, 2000.

8. M. Mendelson, M. Marillier, S. Bailly et al., "Maximal exercise capacity in patients with obstructive sleep apnoea syndrome: A systematic review and meta-analysis," The European respiratory journal, vol. 51, no. 6, 2018.

9. M. Berger, C. E. Kline, F. X. Cepeda et al., "Does obstructive sleep apnea affect exercise capacity and the hemodynamic response to exercise? An individual patient data and aggregate meta-analysis," Sleep medicine reviews, vol. 45, pp. 42-53, 2019.

10. H. V. Fletcher, P. S. Pan Cho, S. Lee Loong et al., "Effect of continuous positive airway pressure on maximal exercise capacity in patients with obstructive sleep apnea: A systematic review and metaanalysis," Journal of clinical sleep medicine : JCSM : official publication of the American Academy of Sleep Medicine, 2020.

11. B. Wåhlin Larsson, F. Kadi, J. Ulfberg et al., "Skeletal muscle morphology and aerobic capacity in patients with obstructive sleep apnoea syndrome," Respiration; international review of thoracic diseases, vol. 76, no. 1, pp. 21-27, 2008.

12. D. Friberg, T. Ansved, K. Borg et al., "Histological indications of a progressive snorers disease in an upper airway muscle," American journal of respiratory and critical care medicine, vol. 157, no. 2, pp. 586-593, 1998.

13. L. Edström, H. Larsson, and L. Larsson, "Neurogenic effects on the palatopharyngeal muscle in patients with obstructive sleep apnoea: A muscle biopsy study," Journal of neurology, neurosurgery, and psychiatry, vol. 55, no. 10, pp. 916-920, 1992.

14. R. Lindman and P. S. Stål, "Abnormal palatopharyngeal muscle morphology in sleep-disordered breathing," Journal of the neurological sciences, vol. 195, no. 1, pp. 11-23, 2002.

15. J. H. Boyd, B. J. Petrof, Q. Hamid et al., "Upper airway muscle inflammation and denervation changes in obstructive sleep apnea," American journal of respiratory and critical care medicine, vol. 170, no. 5, 
pp. 541-546, 2004.

16. C.-P. Ko, "Neuromuscular System," in International Encyclopedia of the Social \& Behavioral Sciences, pp. 10595-10600, Elsevier, 2001.

17. M. H. Andonian and M. A. Fahim, "Endurance exercise alters the morphology of fast- and slow-twitch rat neuromuscular junctions," International journal of sports medicine, vol. 9, no. 3, pp. 218-223, 1988.

18. M. R. Deschenes, C. M. Maresh, J. F. Crivello et al., "The effects of exercise training of different intensities on neuromuscular junction morphology," Journal of neurocytology, vol. 22, no. 8, pp. 603$615,1993$.

19. M. R. Deschenes, D. A. Judelson, W. J. Kraemer et al., "Effects of resistance training on neuromuscular junction morphology," Muscle \& nerve, vol. 23, no. 10, pp. 1576-1581, 2000.

20. M. R. Deschenes, J. Covault, W. J. Kraemer et al., "The neuromuscular junction. Muscle fibre type differences, plasticity and adaptability to increased and decreased activity," Sports medicine (Auckland, N.Z.), vol. 17, no. 6, pp. 358-372, 1994.

21. M. R. Deschenes, S. Li, M. A. Adan et al., "Muscle fibers and their synapses differentially adapt to aging and endurance training," Experimental gerontology, vol. 106, pp. 183-191, 2018.

22. M. R. Deschenes and M. H. Wilson, "Age-related differences in synaptic plasticity following muscle unloading," Journal of neurobiology, vol. 57, no. 3, pp. 246-256, 2003.

23. L. Dupuis, J.-L. Gonzalez de Aguilar, A. Echaniz-Laguna et al., "Muscle mitochondrial uncoupling dismantles neuromuscular junction and triggers distal degeneration of motor neurons," PloS one, vol. 4, no. 4, e5390, 2009.

24. Y. C. Jang and H. van Remmen, "Age-associated alterations of the neuromuscular junction," Experimental gerontology, vol. 46, 2-3, pp. 193-198, 2011.

25. O. A. Mesarwi, E. V. Sharma, J. C. Jun et al., "Metabolic dysfunction in obstructive sleep apnea: A critical examination of underlying mechanisms," Sleep and biological rhythms, vol. 13, no. 1, pp. 217, 2015.

26. J. L. Evans, B. A. Maddux, and I. D. Goldfine, "The molecular basis for oxidative stress-induced insulin resistance," Antioxidants \& redox signaling, vol. 7, 7-8, pp. 1040-1052, 2005.

27. R. Schulz, S. Mahmoudi, K. Hattar et al., "Enhanced release of superoxide from polymorphonuclear neutrophils in obstructive sleep apnea. Impact of continuous positive airway pressure therapy," American journal of respiratory and critical care medicine, vol. 162, 2 Pt 1, pp. 566-570, 2000.

28. H.-J. Eisele, P. Markart, and R. Schulz, "Obstructive Sleep Apnea, Oxidative Stress, and Cardiovascular Disease: Evidence from Human Studies," Oxidative medicine and cellular longevity, vol. 2015, p. $608438,2015$.

29. R. Dumitrascu, J. Heitmann, W. Seeger et al., "Obstructive sleep apnea, oxidative stress and cardiovascular disease: Lessons from animal studies," Oxidative medicine and cellular longevity, vol. 2013, p. 234631, 2013. 
30. H. Greenberg, X. Ye, D. Wilson et al., "Chronic intermittent hypoxia activates nuclear factor-kappaB in cardiovascular tissues in vivo," Biochemical and biophysical research communications, vol. 343, no. 2, pp. 591-596, 2006.

31. K. Kim, "Interaction between HSP 70 and iNOS in skeletal muscle injury and repair," Journal of exercise rehabilitation, vol. 11, no. 5, pp. 240-243, 2015.

32. G. Zhan, P. Fenik, D. Pratico et al., "Inducible nitric oxide synthase in long-term intermittent hypoxia: Hypersomnolence and brain injury," American journal of respiratory and critical care medicine, vol. 171, no. 12, pp. 1414-1420, 2005.

33. F. L. M. Ricciardolo, G. Caramori, K. Ito et al., "Nitrosative stress in the bronchial mucosa of severe chronic obstructive pulmonary disease," The Journal of allergy and clinical immunology, vol. 116, no. 5, pp. 1028-1035, 2005.

34. K. Eghbalzadeh, K. Brixius, W. Bloch et al., "Skeletal muscle nitric oxide (NO) synthases and NOsignaling in "diabesity" - What about the relevance of exercise training interventions?," Nitric Oxide, vol. 37, pp. 28-40, 2014.

35. G. A. Bonaterra, H. Then, L. Oezel et al., "Morphological Alterations in Gastrocnemius and Soleus Muscles in Male and Female Mice in a Fibromyalgia Model," PloS one, vol. 11, no. 3, e0151116, 2016.

36. L. Lavie and P. Lavie, "Molecular mechanisms of cardiovascular disease in OSAHS: The oxidative stress link," The European respiratory journal, vol. 33, no. 6, pp. 1467-1484, 2009.

37. C. Wu, L. Tang, X. Ni et al., "Salidroside Attenuates Denervation-Induced Skeletal Muscle Atrophy Through Negative Regulation of Pro-inflammatory Cytokine," Frontiers in physiology, vol. 10, p. 665, 2019.

38. P. Lebrun, E. Cognard, R. Bellon-Paul et al., "Constitutive expression of suppressor of cytokine signalling-3 in skeletal muscle leads to reduced mobility and overweight in mice," Diabetologia, vol. 52, no. 10, pp. 2201-2212, 2009.

39. B. R. McKay, D. I. Ogborn, J. M. Baker et al., "Elevated SOCS3 and altered IL-6 signaling is associated with age-related human muscle stem cell dysfunction," American Journal of Physiology-Cell Physiology, vol. 304, no. 8, C717-C728, 2013.

40. Z. Yang, M. Hulver, R. P. McMillan et al., "Regulation of Insulin and Leptin Signaling by Muscle Suppressor of Cytokine Signaling 3 (SOCS3)," PloS one, vol. 7, no. 10, e47493, 2012.

41. S. B. Jorgensen, H. M. O'Neill, L. Sylow et al., "Deletion of skeletal muscle SOCS3 prevents insulin resistance in obesity," Diabetes, vol. 62, no. 1, pp. 56-64, 2013.

42. E. Sidlauskaite, J. W. Gibson, I. L. Megson et al., "Mitochondrial ROS cause motor deficits induced by synaptic inactivity: Implications for synapse pruning," Redox biology, vol. 16, pp. 344-351, 2018.

43. T. Y. Kostrominova, K. A. Pasyk, H. van Remmen et al., "Adaptive changes in structure of skeletal muscles from adult Sod1 homozygous knockout mice," Cell and tissue research, vol. 327, no. 3, pp. 595-605, 2007.

44. V. A. Kadhiresan, C. A. Hassett, and J. A. Faulkner, "Properties of single motor units in medial gastrocnemius muscles of adult and old rats," The Journal of physiology, 493 (Pt 2), pp. 543-552, 
1996.

45. J. Nanduri, N. Wang, G. Yuan et al., "Intermittent hypoxia degrades HIF-2alpha via calpains resulting in oxidative stress: Implications for recurrent apnea-induced morbidities," Proceedings of the National Academy of Sciences of the United States of America, vol. 106, no. 4, pp. 1199-1204, 2009.

46. E. Wysocka, S. Cofta, M. Cymerys et al., "The impact of the sleep apnea syndrome on oxidantantioxidant balance in the blood of overweight and obese patients," Journal of physiology and pharmacology : an official journal of the Polish Physiological Society, 59 Suppl 6, pp. 761-769, 2008.

47. J. Sauleda, F. J. García-Palmer, S. Tarraga et al., "Skeletal muscle changes in patients with obstructive sleep apnoea syndrome," Respiratory medicine, vol. 97, no. 7, pp. 804-810, 2003.

48. T. Y. Kostrominova, "Advanced age-related denervation and fiber-type grouping in skeletal muscle of SOD1 knockout mice," Free radical biology \& medicine, vol. 49, no. 10, pp. 1582-1593, 2010.

49. J. P. Charles, O. Cappellari, A. J. Spence et al., "Musculoskeletal Geometry, Muscle Architecture and Functional Specialisations of the Mouse Hindlimb," PloS one, vol. 11, no. 4, e0147669, 2016.

50. M. R. Deschenes, M. A. Roby, and E. K. Glass, "Aging influences adaptations of the neuromuscular junction to endurance training," Neuroscience, vol. 190, pp. 56-66, 2011.

51. S. Willadt, M. Nash, and C. Slater, "Age-related changes in the structure and function of mammalian neuromuscular junctions," Annals of the New York Academy of Sciences, vol. 1412, no. 1, pp. 41-53, 2018.

52. G. Valdez, J. C. Tapia, J. W. Lichtman et al., "Shared resistance to aging and ALS in neuromuscular junctions of specific muscles," PloS one, vol. 7, no. 4, e34640, 2012.

53. P. S. Stål and B. Johansson, "Abnormal mitochondria organization and oxidative activity in the palate muscles of long-term snorers with obstructive sleep apnea," Respiration; international review of thoracic diseases, vol. 83, no. 5, pp. 407-417, 2012.

54. R. J. Wilson, J. C. Drake, Di Cui et al., "Mitochondrial protein S-nitrosation protects against ischemia reperfusion-induced denervation at neuromuscular junction in skeletal muscle," Free radical biology \& medicine, vol. 117, pp. 180-190, 2018.

55. R. Schulz, G. Murzabekova, B. Egemnazarov et al., "Arterial hypertension in a murine model of sleep apnea: Role of NADPH oxidase 2," Journal of hypertension, vol. 32, no. 2, pp. 300-305, 2014.

56. A. N. Billin, S. E. Honeycutt, A. V. McDougal et al., "HIF prolyl hydroxylase inhibition protects skeletal muscle from eccentric contraction-induced injury," Skeletal muscle, vol. 8, no. 1, p. 35, 2018.

57. D. Levy, P. Kubes, and D. W. Zochodne, "Delayed peripheral Nerve Degeneration, Regeneration, and pain in Mice Lacking Inducible Nitric Oxide Synthase," Journal of Neuropathology \& Experimental Neurology, vol. 60, no. 5, pp. 411-421, 2001.

58. P. Mayer, M. Dematteis, J. L. Pépin et al., "Peripheral neuropathy in sleep apnea. A tissue marker of the severity of nocturnal desaturation," American journal of respiratory and critical care medicine, vol. 159, no. 1, pp. 213-219, 1999. 
59. S. Ramchandren, K. L. Gruis, R. D. Chervin et al., "Hypoglossal nerve conduction findings in obstructive sleep apnea," Muscle \& nerve, vol. 42, no. 2, pp. 257-261, 2010.

60. D. J. Eckert, J. P. Saboisky, A. S. Jordan et al., "Upper airway myopathy is not important in the pathophysiology of obstructive sleep apnea," Journal of clinical sleep medicine : JCSM : official publication of the American Academy of Sleep Medicine, vol. 3, no. 6, pp. 570-573, 2007.

61. A. Cheng, M. Morsch, Y. Murata et al., "Sequence of age-associated changes to the mouse neuromuscular junction and the protective effects of voluntary exercise," PloS one, vol. 8, no. 7, e67970, 2013.

62. W. Liu, L. Wei-LaPierre, A. Klose et al., "Inducible depletion of adult skeletal muscle stem cells impairs the regeneration of neuromuscular junctions," eL ife, vol. 4, 2015.

63. K. Flurkey, J. M. Currer, and D. E. Harrison, "Mouse Models in Aging Research," in The mouse in biomedical research, J. G. Fox, M. T. Davisson, F. W. Quimby et al., Eds., III, pp. 637-672, Elsevier Academic Press, Amsterdam, 2007.

64. K. Hemmrich, C. V. Suschek, G. Lerzynski et al., "iNOS activity is essential for endothelial stress gene expression protecting against oxidative damage," Journal of applied physiology (Bethesda, Md. : 1985), vol. 95, no. 5, pp. 1937-1946, 2003.

65. R. B. Mikkelsen and P. Wardman, "Biological chemistry of reactive oxygen and nitrogen and radiationinduced signal transduction mechanisms," Oncogene, vol. 22, no. 37, pp. 5734-5754, 2003.

66. H. Bayir, V. E. Kagan, G. G. Borisenko et al., "Enhanced oxidative stress in iNOS-deficient mice after traumatic brain injury: Support for a neuroprotective role of iNOS," Journal of cerebral blood flow and metabolism : official journal of the International Society of Cerebral Blood Flow and Metabolism, vol. 25, no. 6, pp. 673-684, 2005.

67. M. P. Cole, L. Chaiswing, T. D. Oberley et al., "The protective roles of nitric oxide and superoxide dismutase in adriamycin-induced cardiotoxicity," Cardiovascular research, vol. 69, no. 1, pp. 186197, 2006.

68. L. Chaiswing, M. P. Cole, W. Ittarat et al., "Manganese superoxide dismutase and inducible nitric oxide synthase modify early oxidative events in acute adriamycin-induced mitochondrial toxicity," Molecular cancer therapeutics, vol. 4, no. 7, pp. 1056-1064, 2005.

\section{Figures}



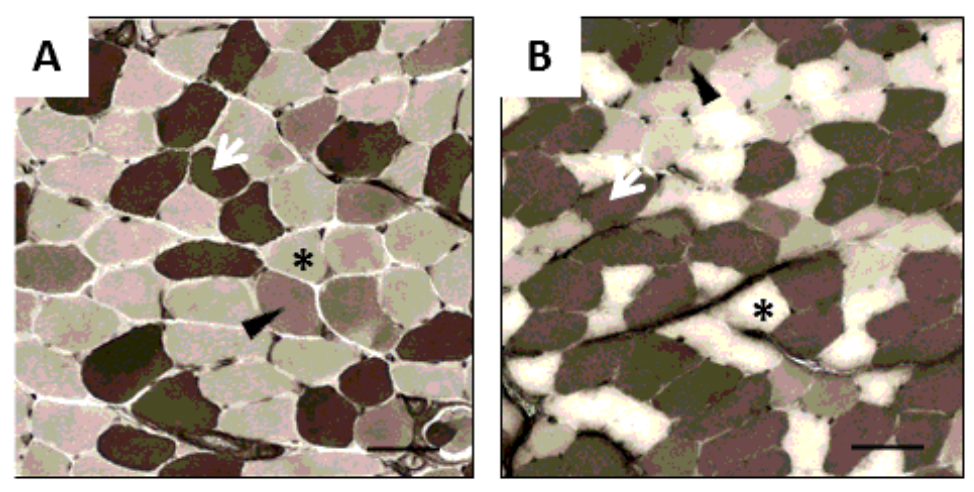

E
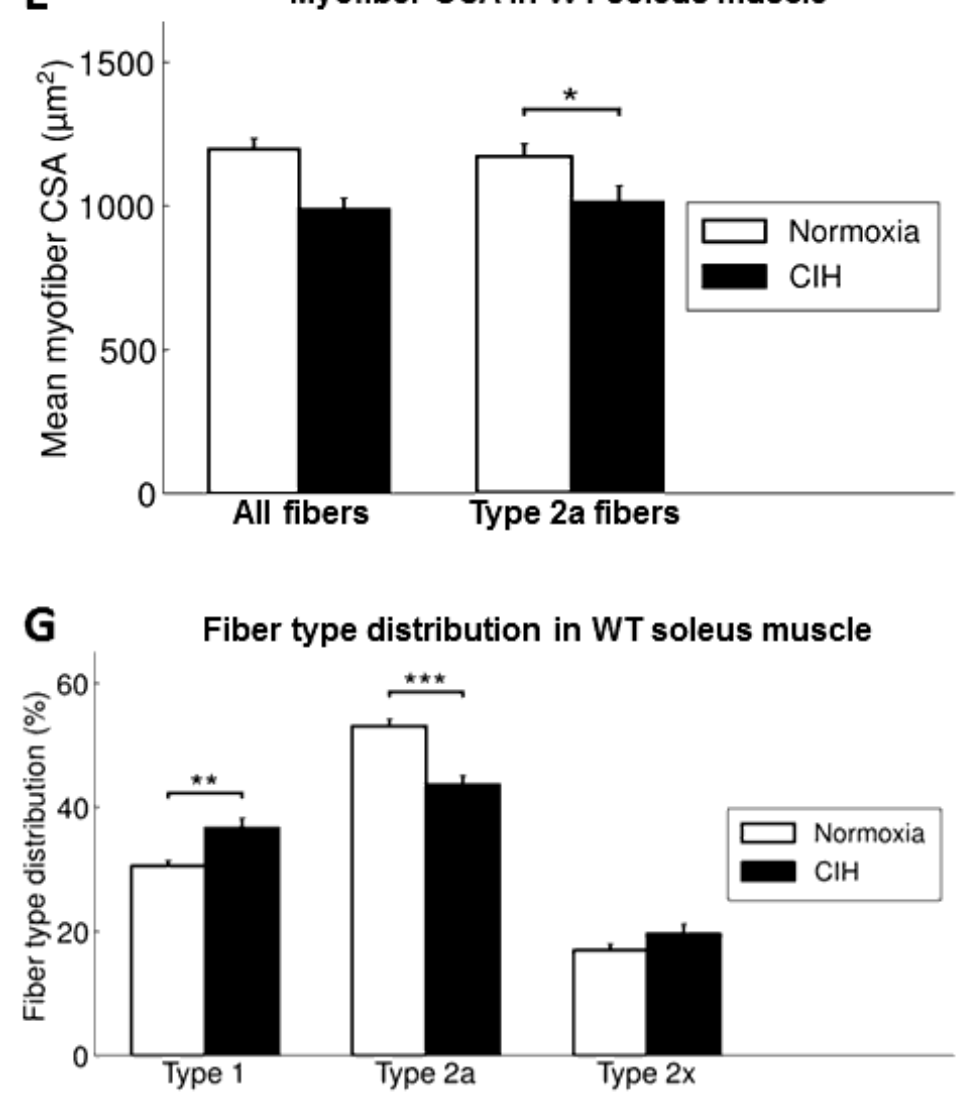
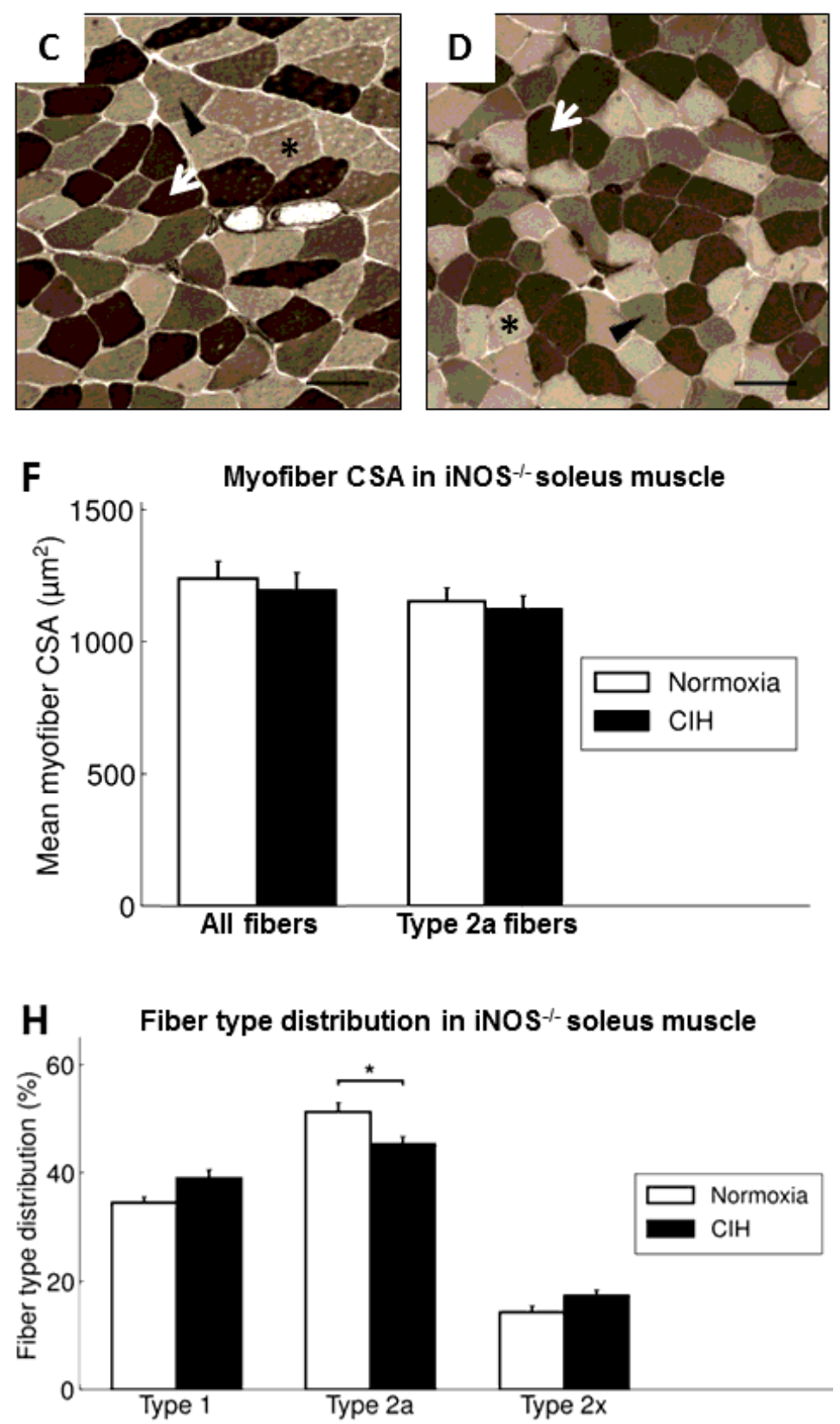

\section{Figure 1}

Representative images (200fold magnification, scale bar $=50 \mu \mathrm{m}$ ) of soleus muscle cross sections stained for ATPase after preincubation at pH 4.55 of WT-NOX mouse (A), WT-ClH mouse (B), iNOS-/-NOX mouse (C) and iNOS-/-ClH mouse (D). Type 1 fibers are stained dark (white arrow), type 2a fibers are stained light-coloured (black star), and type $2 x$ fibers are stained intermediate (black arrow head). (E) Overall myofiber CSA [ $\left.\mu \mathrm{m}^{2}\right]$ and $(\mathrm{F})$ CSA of type 2a myofibers in soleus muscle of WT and iNOS-/-mice. Fiber type distribution [\%] in soleus muscle. Percentage of type 1, type $2 a$ and type $2 x$ fibers of WT mice after 6 weeks of $\mathrm{ClH}$ compared with NOX controls $(G)$ and of iNOS-/- mice $(H)$. Values are given as mean+SEM; $n=8-14$ animals per group. ${ }^{*} p<0.05,{ }^{* \star} p<0.01,{ }^{\star \star *} p<0.001$, significance between $\mathrm{CIH}$ and NOX. 


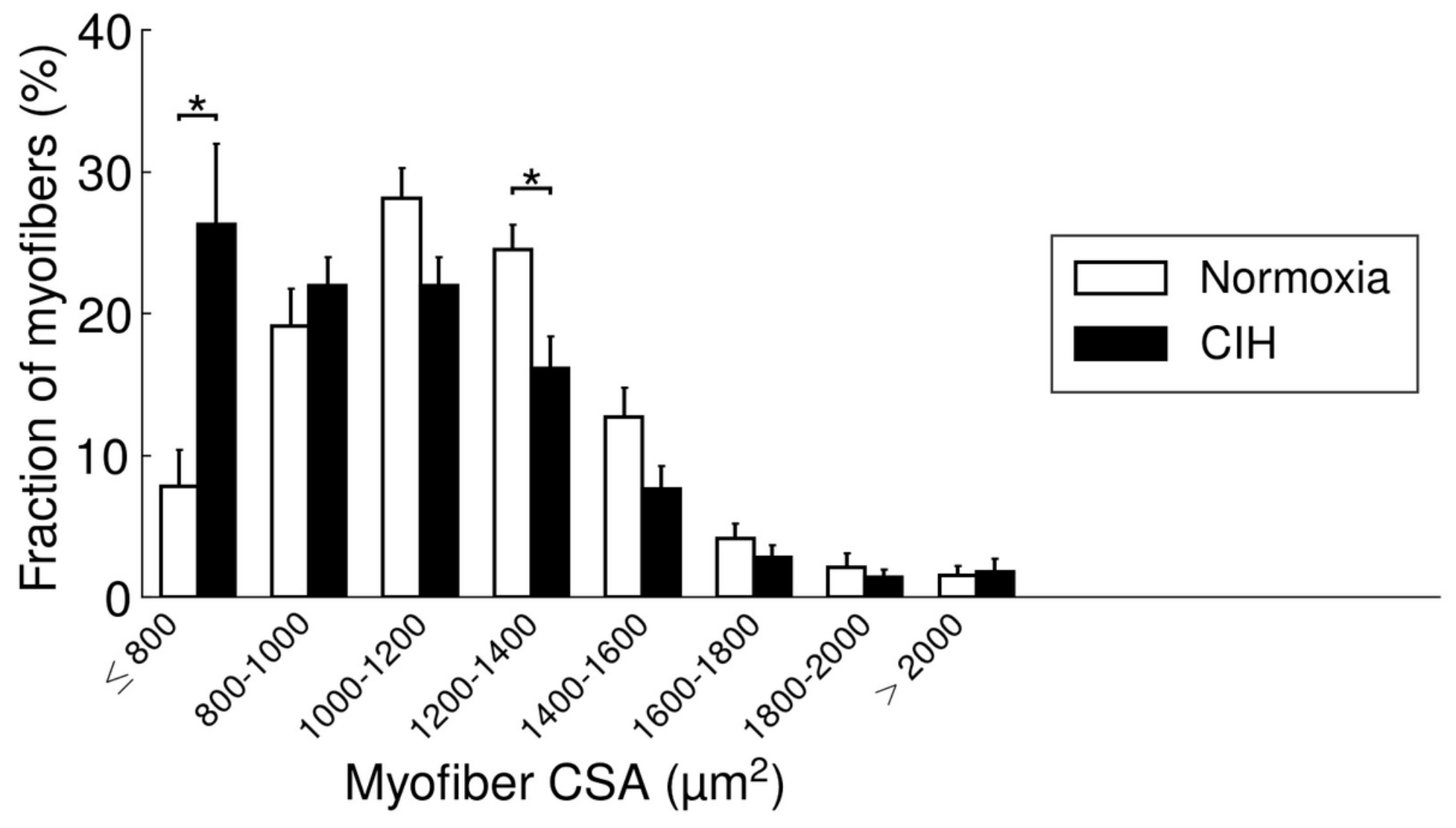

Figure 2

Histogram of myofiber CSA in soleus muscle in WT mice. Values are given as mean+SEM; $n=12-14$ animals per group. * $p<0.05$, significance between WT-CIH and WT-NOX. 


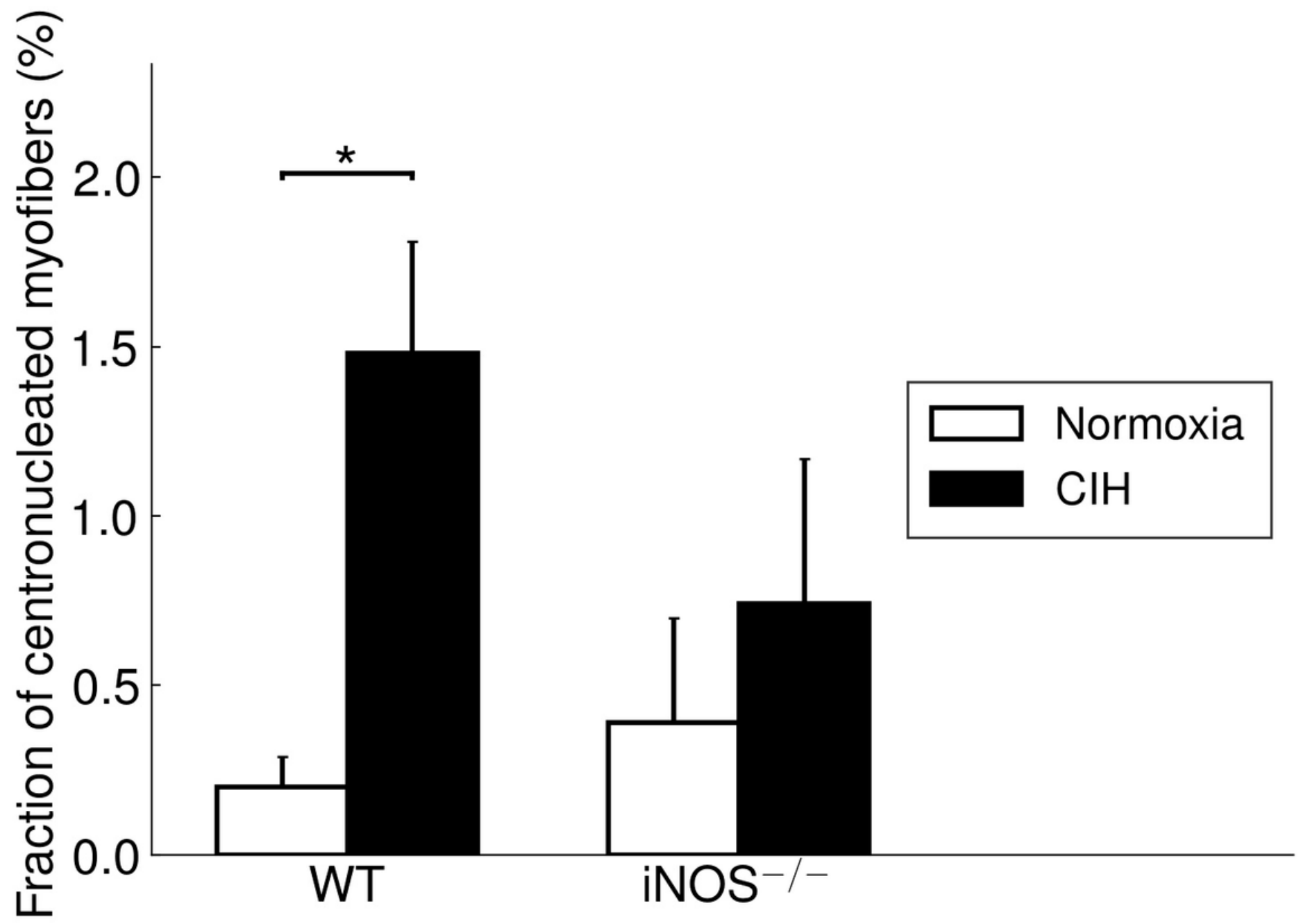

Figure 3

The percentage of centronucleated fibers in soleus muscle is shown. Values are given as mean+SEM; $n=8-14$ animals per group. ${ }^{*} p<0.05$, significance between $\mathrm{ClH}$ and NOX. 

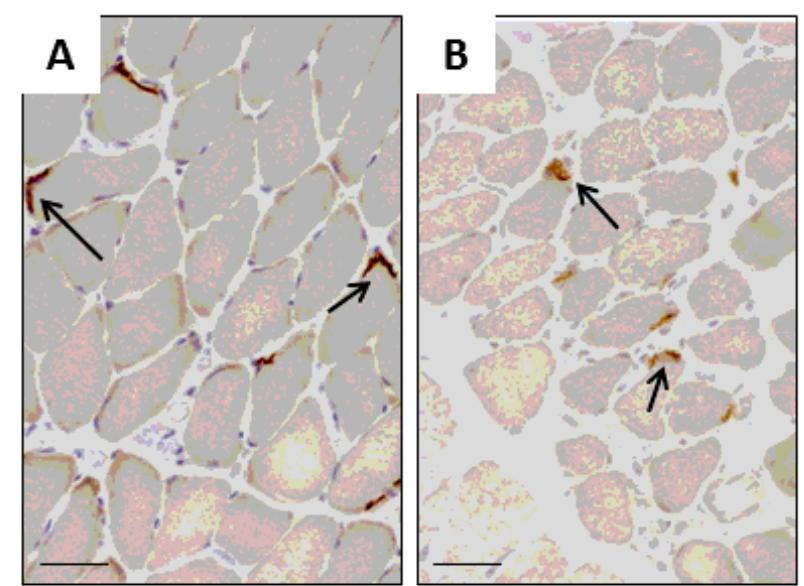

D

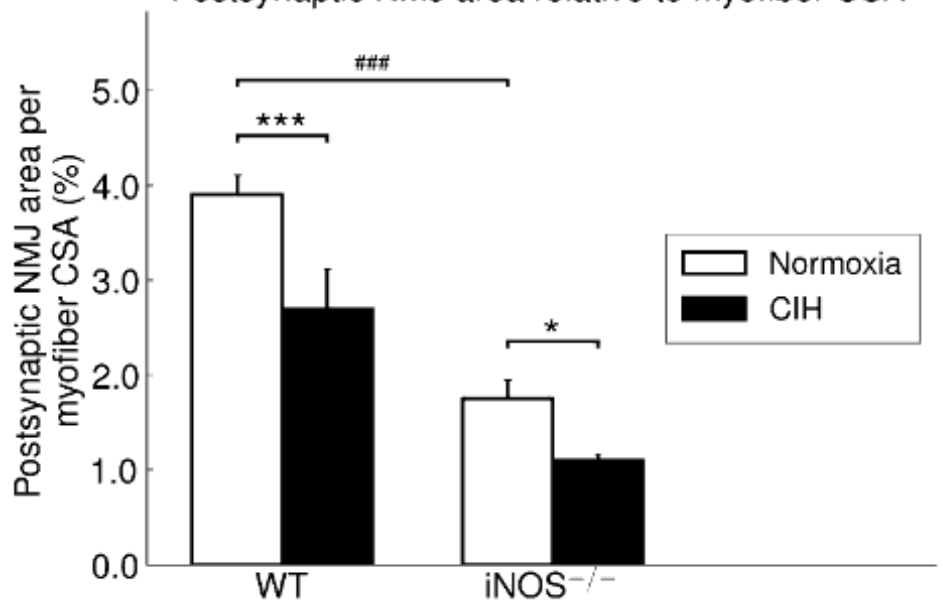

$\mathbf{F}$

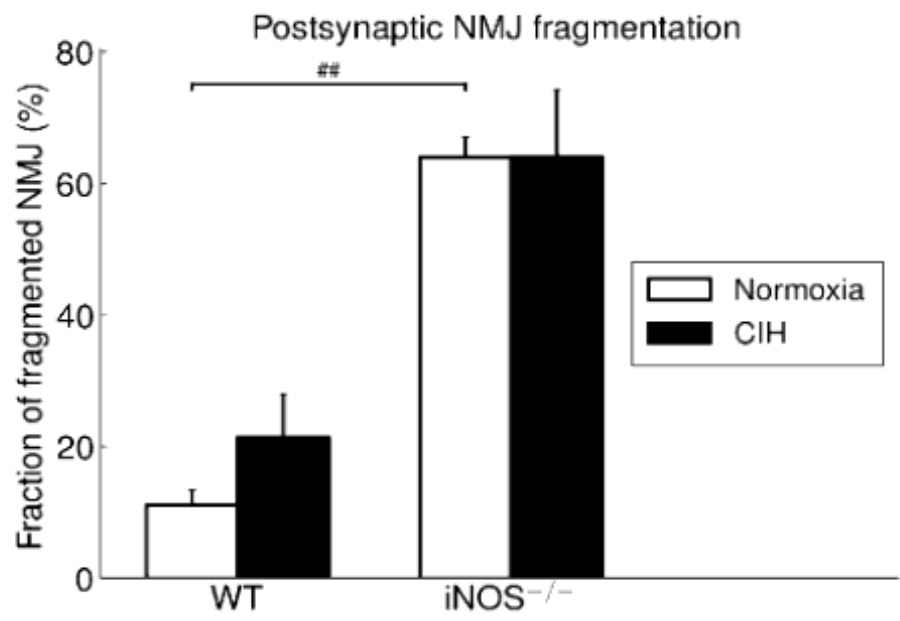

C

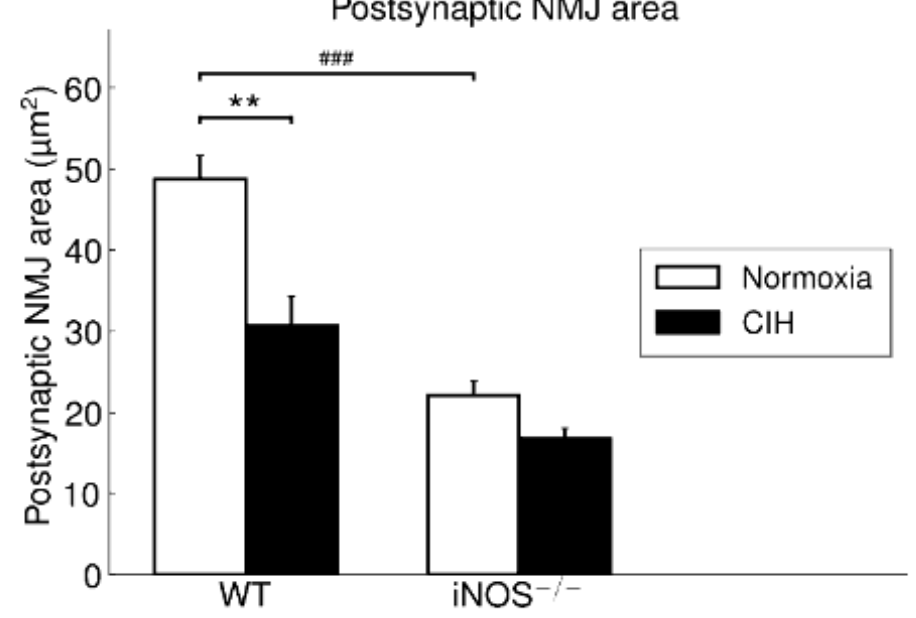

$\mathrm{E}$
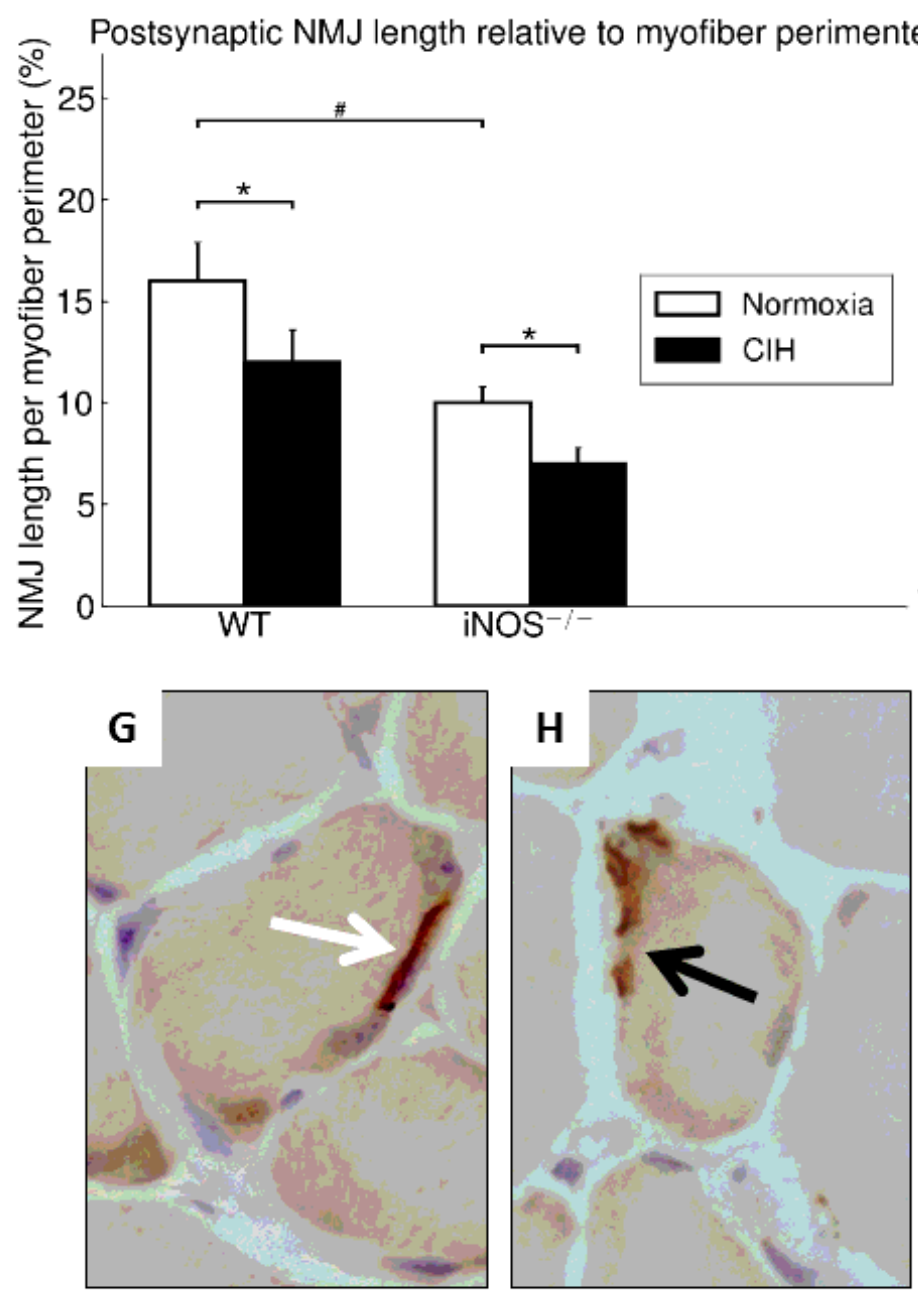

Figure 4

Representative images (200fold magnification, scale bar $=50 \mu \mathrm{m}$ ) of soleus muscle cross sections stained for BTX of WT-NOX (A), WT-CIH (B) are shown. Postsynaptic NMJ area in soleus muscle (C). Postsynaptic NMJ area normalized to myofiber CSA (D) and NMJ length relative to myofiber perimeter (E) as well as percentage of fragmented NMJ in soleus muscle (F). Representative images (400fold magnification) of BTX-stained AChR distribution at muscular NMJ in soleus muscle (G-H). (G) Postsynaptic BTX-stained 
NMJ from WT-NOX mouse (white arrow). (H) Fragmented postsynaptic BTX-stained NMJ of WT-CIH mouse is (black arrow). Values are given as mean+SEM; $n=8$ animals per group. * $p<0.05, * \star p<0.01, * \star \star$ $\mathrm{p}<0.001$, significance between $\mathrm{CIH}$ and NOX; \# $\mathrm{p}<0.05, \# \# \mathrm{p}<0.01$, \#\#\# $\mathrm{p}<0.001$, significance between WT and iNOS-/-.

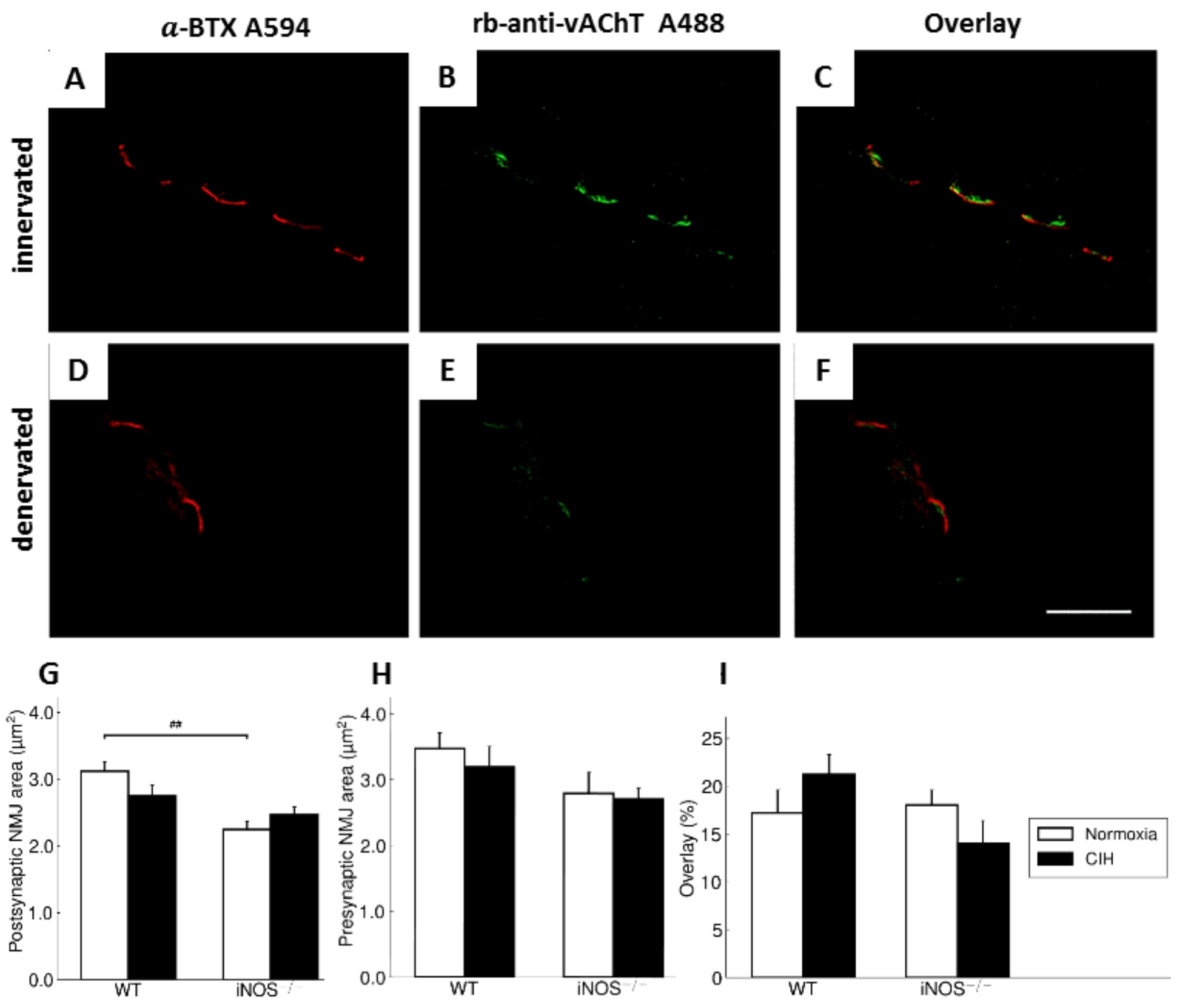

\section{Figure 5}

Confocal projection images of representative NMJ from the vastus muscle stained for postsynaptic AChR $(B T X, A+D$, red) and for nerve terminal vAChT (B+E, green). The merge is showing the colocalisation of $\mathrm{BTX}$ and vAChT $(\mathrm{C}+\mathrm{F})$. Example is given of an innervated muscular NMJ where the BTX-stained area is largely covered by the $\mathrm{AAChT}$ staining $(\mathrm{A}-\mathrm{C})$. In contrast, $\mathrm{D}-\mathrm{F}$ are showing a NMJ where the AChRstained areas are only partially covered by vAChT staining, representing denervation. Scale bar in panel $F$ is $10 \mu \mathrm{m}$. Mean postsynaptic $(\mathrm{G})$ and presynaptic $(\mathrm{H}) \mathrm{NMJ}$ areas and overlay areas of BTX and vAChT (I) 
in vastus muscle are shown. Values are given as mean+SEM; $n=4-6$ animals per group. \#\# $p<0.01$, significance between WT and iNOS-/-.
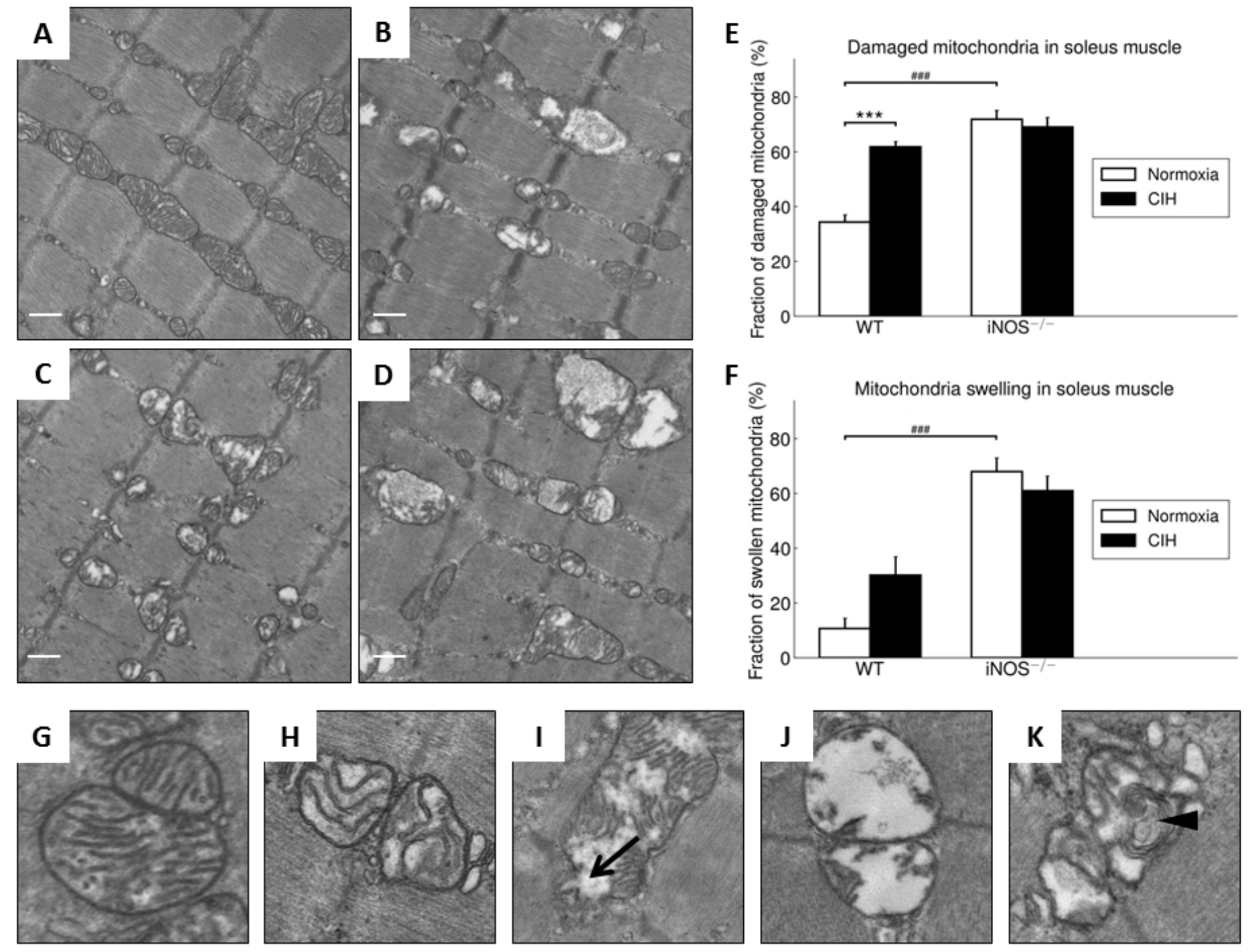

\section{Figure 6}

Representative TEM images of WT (A, B) and iNOS-/- (C, D) soleus muscle under CIH (B, D) vs. NOX (A, C) (scale bars $=100 \mathrm{~nm}$ ). Percentage of damaged (E) and swollen mitochondria (F) are shown. Mitochondria presented various morphological abnormalities such as outer membrane rupture (l; black arrow), loss of cristae $(\mathrm{J})$ or multi-lammelar bodies (K; black arrow head). $\mathrm{G}$ and $\mathrm{H}$ correspond to intact mitochondria completely $(\mathrm{G})$ or partially $(\mathrm{H})$ filled with cristae. Values are given as mean+SEM, $n=7-9$ animals per group. *** $p<0.001$, significance between $\mathrm{CIH}$ and NOX, \#\#\# $\mathrm{p}<0.001$, significance between WT and iNOS-/-.

\section{Supplementary Files}

This is a list of supplementary files associated with this preprint. Click to download. 
- additionalfile2.jpg

- additionalfile3.jpg

- additionalfile4.jpg

- additionalfile5.jpg

- additionalfile6.jpg

- tableadditional.docx 